\title{
Ginsenoside Rb1 inhibits free fatty acids-induced oxidative stress and inflammation in 3T3-L1 adipocytes
}

\author{
MIN WANG $^{1 *}$, YANMING CHEN ${ }^{2 *}$, ZHAOJUN XIONG $^{1}$, SHUJIE YU $^{1}$, BIN ZHOU $^{1}$, YESHENG LING $^{1}$, \\ ZHENDA ZHENG $^{1}$, GUANGYAO SHI ${ }^{1}$, YONGXIANG WU ${ }^{1}$ and XIAOXIAN QIAN ${ }^{1}$ \\ Departments of ${ }^{1}$ Cardiology and ${ }^{2}$ Endocrinology, The Third Affiliated Hospital of \\ Sun Yat-sen University, Guangzhou, Guangdong 510630, P.R. China
}

Received February 26, 2017; Accepted August 14, 2017

DOI: $10.3892 / \mathrm{mmr} .2017 .7710$

\begin{abstract}
Free fatty acids (FFAs) increase in visceral fat and are inferred to be one of the underlying inducers of adipose tissue inflammation. In our previous study, it was demonstrated that ginsenoside Rb1 stimulates endothelial nitric oxide synthase (eNOS) and Sirtuin 1 to protect against endothelial cell senescence. In the present study, 3T3-L1 adipocytes were exposed to $0.5 \mathrm{mM}$ FFAs with or without Rb1 (10-40 $\mu \mathrm{M})$. Monocyte chemotactic protein-1 (MCP-1) and interleukin-6 (IL-6) secretion was measured using ELISA. Tumor necrosis factor- $\alpha(\mathrm{TNF}-\alpha)$ expression and nuclear factor $-\kappa \mathrm{B}(\mathrm{NF}-\kappa \mathrm{B})$ p65 phosphorylation were detected using western blot analysis. Oxidative stress was determined via measuring intracellular reactive oxygen species (ROS) and nitric oxide (NO) production. The results demonstrated that MCP-1 and IL-6 secretion, as well as TNF- $\alpha$ expression, were significantly increased following FFA treatment, which was attenuated by Rb1 in a dose-dependent manner. Furthermore, Rb1 attenuated FFA-induced $\mathrm{NF}-\kappa \mathrm{B}$ phosphorylation, suggesting that the inhibitory effect of Rb1 on inflammatory cytokines was partially mediated through blockade of NF- $\kappa \mathrm{B}$ phosphorylation. Further experiments demonstrated that Rb1 ameliorated FFA-induced ROS generation and NO reduction through upregulation of superoxide dismutase 2 and eNOS expression. Taken together, these results demonstrate proinflammatory and pro-oxidant effects of FFA on 3T3-L1 adipocytes, which are effectively ameliorated by Rb1. Suppression of inflammatory responses and oxidative stress may be a novel mechanism for attenuating the effect of Rb1 on adipocyte dysfunction.
\end{abstract}

Correspondence to: Professor Xiaoxian Qian, Department of Cardiology, The Third Affiliated Hospital of Sun Yat-sen University, 600 Tianhe Road, Guangzhou, Guangdong 510630, P.R. China E-mail: yyqianxiaoxian@163.com

\section{${ }^{*}$ Contributed equally}

Key words: ginsenoside Rb1, 3T3-L1 adipocytes, oxidative stress, free fatty acid

\section{Introduction}

Obesity has been identified as a kind of chronic low-grade inflammatory condition and is closely associated with the development of insulin resistance, type 2 diabetes, cardiovascular disease, and cancer (1-3). Adipose tissue, which contains diverse types of cells including pre-adipocytes, adipocytes, endothelial cells, and immune cells, has recently been identified as a pivotal endocrine tissue (4,5). A number of recent studies have shown that adipocytes synthesize and secrete a large amount of hormones, and inflammatory cytokines into systemic circulation, including adiponectin, leptin, tumor necrosis factor- $\alpha$ (TNF- $\alpha)$, monocyte chemoattractant protein 1 (MCP-1), interleukin-6 (IL-6) and plasminogen activator inhibitor-1 (6-8). In the obese state, most free fatty acids (FFAs) are derived from adipose tissue, which stimulate adipocytes to release pro-inflammatory cytokines and contribute to development of the inflammatory state and oxidative stress $(9,10)$. FFAs mediate these responses in part through activation of the nuclear factor- $\kappa \mathrm{B}(\mathrm{NF}-\kappa \mathrm{B})$ pathway, which activate abundant secretion of inflammatory cytokines and inhibit insulin signaling (11). In addition, FFAs are implicated in the activation of oxidative stress partly by impairment of endogenous antioxidant defenses.

Asian ginseng, the root of Panax ginseng C.A. Meyer (Araliaceae), is a widely used herbal medicine in East Asia. Ginsenosides, the major pharmacologically active ingredients of ginseng, appear to provide an effective therapy for neurodegenerative diseases (12) and inhibit inflammation, redox stress (13), and cellular senescence. Ginsenosides are generally divided into two groups, panaxadiols and panaxatriols, based on their chemical structure. Panaxadiols include compounds such as the ginsenoside Rb1, the most abundant among more than 40 ginsenosides. Rb1 has been extensively studied and found to have multiple biological functions including anti-inflammation, anti-apoptosis, anti-oxidation, increasing nitric oxide production in endothelial cells, and inhibiting angiogenesis.

Recent studies have found that Rb1 improves insulin sensitivity in obese and diabetic $\mathrm{db} / \mathrm{db}$ mice by reducing hepatic fat accumulation and suppressing adipocyte lipolysis via up-regulation of perilipin expression in adipocytes (14). Another important finding is that Rb1 has anti-obesity and anti-hyperglycemic 
effects in diet-induced obese rats (15). Our recent study demonstrated that Rb1 pretreatment prevents human umbilical vein endothelial cell (HUVEC) senescence through modulation of the redox status and protects HUVECs from hydrogen peroxide $\left(\mathrm{H}_{2} \mathrm{O}_{2}\right)$-induced senescence through stimulation of the Sirtuin 1 pathway (16). However, limited data have been reported concerning the effect of Rb1 on FFA-induced inflammation in adipocytes. In this study, we investigated whether Rb1 inhibits inflammatory responses induced by FFAs in 3T3-L1 adipocytes and the underlying mechanism.

\section{Materials and methods}

Cell culture and treatments. Mouse embryonic 3T3-L1 pre-adipocytes were purchased from the American Type Culture Collection (Manassas, VA, USA) and maintained in high-glucose Dulbecco's modified Eagle's medium (DMEM) (Invitrogen, Carlsbad, CA, USA) supplemented with $10 \%$ bovine calf serum (Hyclone, Logan, UT, USA) at $37^{\circ} \mathrm{C}$ in a $5 \% \mathrm{CO}_{2}$ incubator until confluency and then induced to differentiate as described previously $(17,18)$. Briefly, at 2 days post-confluency (defined as day 0), the cells were exposed to differentiation medium containing $0.5 \mathrm{mM}$ isobutylmethylxanthine, $1 \mathrm{M}$ dexamethasone, $10 \mu \mathrm{g} / \mathrm{ml}$ insulin (Sigma, St. Louis, MO, USA), and 10\% fetal bovine serum (FBS) for 3 days. Then, the cells were transferred to DMEM with $10 \mu \mathrm{g} / \mathrm{ml}$ insulin and $10 \%$ FBS. The medium was changed every two days. Maturation of adipocytes was confirmed by Oil red staining. Differentiated adipocytes were serum starved for $16 \mathrm{~h}$ in DMEM supplemented with $2 \%$ FBS before treatment and then exposed to FFAs for $4 \mathrm{~h}$. For Rb1 treatments, 3T3-L1 adipocytes were treated with various concentrations of $\mathrm{Rb} 1(10-40 \mu \mathrm{mol} / \mathrm{l})$ for $4 \mathrm{~h}$, followed by treatment with $0.5 \mathrm{mM}$ FFAs for $4 \mathrm{~h}$. At the end of experiments, the culture supernatants and monolayered cells were harvested for analysis.

Preparation of fatty acid-albumin complexes. Saturated palmitic acid was used in this study as FFA. Lipid-containing media were prepared by conjugation of palmitic acids with bovine serum albumin (BSA) using a modified method described by Svedberg et al (19). Briefly, palmitic acids were first dissolved in ethanol at $200 \mathrm{mmol} / \mathrm{l}$ and then combined with $10 \%$ FFA-free low endotoxin BSA to concentrations of 1-10 mmol/l. The $\mathrm{pH}$ of all solutions was adjusted to 7.5 , and the stock solutions were filter sterilized and stored at $-20^{\circ} \mathrm{C}$. A control solution containing ethanol and BSA was prepared similarly. Fresh working solutions were prepared by diluting stock solutions (1:10) in 2\% FBS/endothelial cell basic medium (EBM) or $0.5 \%$ FCS/EBM as appropriate. The final $1 \%$ BSA was consistent in all FFA media, while the FFA-to-BSA ratio varied with the FFA concentration.

Cell viability assay. MTT assay was performed to test viability of 3T3-L1 adipocytes. 3T3-L1 preadipocytes were seeded at a density of $1 \times 10^{4}$ cells per well in a gelatin-coated 96-well plate. After differentiated to mature adipocytes, the cells were then treated with various doses of palmitate $(0,0.125,0.25$, $0.5,1.0 \mathrm{mM}$ ) for $4 \mathrm{~h}$ or $24 \mathrm{~h}$. The cells were used for determination of viability, using the MTT Cell Proliferation Assay kit (Beyotime). Briefly, at indicated time point, the cells were incubated with an MTT solution for $4 \mathrm{~h}$ at $37^{\circ} \mathrm{C}$ in the dark. After supernatants were aspirated, DMSO was added and the plates were agitated to dissolve the formazan crystal product. Absorbance was then measured at $570 \mathrm{~nm}$ in a Victor microplate reader. The percentage of viable cells was calculated by defining the cell viability of control group as $100 \%$.

Measurement of MCP-1 and IL-6 secretion by enzyme-linked immunosorbent assays (ELISAs). Culture supernatants were diluted 2-fold to determine MCP-1 and IL-6 levels. ELISAs were performed according to the manufacturer's instructions (R\&D Systems, Wiesbaden-Nordenstadt, Germany). Briefly, the culture supernatant was collected after treatment and centrifuged to remove any debris. Assay Diluent $(50 \mu \mathrm{l})$ were add to each well, and then standards or sample (50 $\mu \mathrm{l} /$ well) were added to the antibody pre-coated microtiter plates, followed by incubation for $2 \mathrm{~h}$ at room temperature. Then, $100 \mu 1$ MCP-1/IL-6 conjugate were added to each well, followed by incubation for $2 \mathrm{~h}$ at room temperature. After four washes, $100 \mu$ l Substrate Solution were added to each well, followed by incubation for $30 \mathrm{~min}$ while protected from light at room temperature. Stop Solution $(100 \mu \mathrm{l})$ were then added, followed by incubation for less than $30 \mathrm{~min}$. The plate was read immediately at $450 \mathrm{~nm}$ with 540 or $570 \mathrm{~nm}$ as reference wavelengths in a Victor microplate reader. MCP-1 and IL-6 concentrations were calculated according to the standard curve and normalized to the cell numbers.

Western blot analysis. 3T3-L1 preadipocytes were grown and differentiated into adipocytes in six-well plates. After serum starvation in 2\% FBS/DMEM overnight, the cells were incubated in 2\% FBS/DMEM containing 10, 20, or $40 \mathrm{mmol} / 1$ $\mathrm{Rb} 1$ for $4 \mathrm{~h}$, Then, $0.5 \mathrm{mM}$ palmitic acid was added to treated groups for $4 \mathrm{~h}$. Cells were washed twice with precooled PBS and then lysed in RIPA buffer with a protease inhibitor cocktail, PMSF, and sodium orthovanadate (Santa Cruz Biotechnology, Santa Cruz, CA). The protein concentration was measured by the Bradford method. Thirty micrograms of protein in $30 \mu \mathrm{l}$ reducing sample buffer was boiled for $5 \mathrm{~min}$ at $100^{\circ} \mathrm{C}$ and then resolved by sodium dodecyl sulfate-polyacrylamide gel electrophoresis for $2 \mathrm{~h}$ at $100 \mathrm{~V}$. Then, the proteins were transferred onto a polyvinylidene difluoride membrane for $90 \mathrm{~min}$ at $100 \mathrm{~V}$. After transfer, the membrane was incubated in $25 \mathrm{ml}$ blocking buffer (1xTBS, $0.1 \%$ Tween-20 with 5\% non-fat dry milk) for $1 \mathrm{~h}$ at room temperature. Then, the membrane was incubated with primary antibodies against TNF- $\alpha$, endothelial nitric oxide synthase (eNOS), superoxide dismutase 2 (SOD2), phospho-NF- $\kappa$ B (Ser536), NF- $\kappa$ B, or $\beta$-actin (Cell Signaling Technologies, Danvers, MA, USA) in $10 \mathrm{ml}$ primary antibody dilution buffer with gentle agitation overnight at $4^{\circ} \mathrm{C}$. After washing three times for $10 \mathrm{~min}$ each with $15 \mathrm{ml} 10 \mathrm{X} \mathrm{TBS} / 0.1 \%$ TBS/T, the membrane was incubated with a horseradish peroxidase-conjugated secondary antibody (1:3,000; Cell Signaling Technologies, Danvers, MA) in $10 \mathrm{ml}$ blocking buffer with gentle agitation for $1 \mathrm{~h}$ at room temperature, followed by three washes for $10 \mathrm{~min}$ each.

Nitric oxide $(\mathrm{NO})$ production measurement. NO production was evaluated by measuring the accumulation of nitrites, a stable oxidative end product of NO metabolism, in the cell lysate 
of cultured 3T3-L1 adipocytes using the Greiss reagent kit (Beyotime) following the manufacturer's instructions. Briefly, $50 \mu \mathrm{l}$ samples were incubated with $50 \mu \mathrm{l}$ Greiss reagent I and $50 \mu \mathrm{l}$ Greiss reagent II in a 96-well microplate at room temperature for $30 \mathrm{~min}$. The optical density was measured with the Victor microplate reader at $540 \mathrm{~nm}$. Nitrite concentrations in the cell lysates were calculated according to the standard curve.

Determination of reactive oxygen species (ROS). Differentiated 3T3-L1 adipocytes were exposed to $0.5 \mathrm{mM}$ BSA or FFA with or without Rb1 for $4 \mathrm{~h}$ after serum starvation for $16 \mathrm{~h}$. The generation of intracellular ROS was detected by the DCF method using a ROS assay kit (Beyotime). Briefly, treated cells were washed in PBS and then incubated with $10 \mu \mathrm{M}$ 2', 7'-dichlorodihydrofluorescein diacetate in PBS at $37^{\circ} \mathrm{C}$ for $20 \mathrm{~min}$. Fluorescence was measured with excitation/emission wavelengths of 493/538 nm using a fluorescence microscope (DM4000B, Leica, Solms, Germany).

Statistical analysis. Data were calculated and expressed as group means \pm standard deviation. Statistical analyses were performed using the Student's t-test, analysis of variance (ANOVA), and Bonferroni's multiple comparison test. Statistical differences were considered significant at $\mathrm{P}<0.05$.

\section{Results}

FFA induces IL-6 and MCP-1 secretion as well as TNF- $\alpha$ expression in 3T3-L1 adipocytes, and Rbl inhibits these effects. FFAs are a major inducer of the pro-inflammatory response in 3T3-L1 adipocytes and play a central role in obesity. However, the effect of Rb1 on FFA-induced MCP-1 and IL-6 secretion, as well as TNF- $\alpha$ expression is unknown. In the present study, we determined MCP-1 and IL- 6 secretion induced by FFAs using ELISAs and detected the TNF- $\alpha$ protein abundance with an anti-TNF- $\alpha$ antibody by immunoblotting in 3T3-L1 adipocytes. Preliminary experiments using the MTT assay confirmed that incubating 3T3-L1 adipocytes with $1 \mathrm{mM}$ FFAs for $4 \mathrm{~h}$ as well as $24 \mathrm{~h}$ resulted in excessive toxicity whereas $0.5 \mathrm{mM}$ FFA for $4 \mathrm{~h}$ did not affect 3T3-L1 adipocytes viability (Fig. 1). Therefore, the maximum concentration of FFA used in all subsequent experiments was $0.5 \mathrm{mM}$ for $4 \mathrm{~h}$. Differentiated 3T3-L1 adipocytes were exposed to $0.5 \mathrm{mM}$ BSA or $0.5 \mathrm{mM}$ FFAs for $4 \mathrm{~h}$. The results showed that no significant difference was observed between cells exposed to BSA and the normal control, whereas IL-6 (Fig. 2A) and MCP-1 (Fig. 2B) in the medium and TNF- $\alpha$ protein expression (Fig. $2 \mathrm{C}$ ) exposed to FFAs were significantly elevated compared with those exposed to BSA or the normal control (Fig. 2, P<0.01, ANOVA). To determine the effect of Rb1 on FFA-induced MCP-1 and IL-6 production and the TNF- $\alpha$ protein level in 3T3-L1 adipocytes, we exposed cultured 3T3-L1 adipocytes to $0.5 \mathrm{mM}$ FFAs with or without $\mathrm{Rb} 1$ at 10,20 , and $40 \mu \mathrm{M}$ for $4 \mathrm{~h}$. The results showed that Rb1 significantly decreased IL-6 (Fig. 2A) and MCP-1 (Fig. 2B) production as well as TNF- $\alpha$ expression (Fig. 2C) in a dose-dependent manner (Fig. 2, P<0.05, ANOVA).

FFA decreases eNOS expression and NO production in 3T3-L1 adipocytes, and Rbl blocks this effect. NO has been recognized as a potential mediator of inflammation-induced

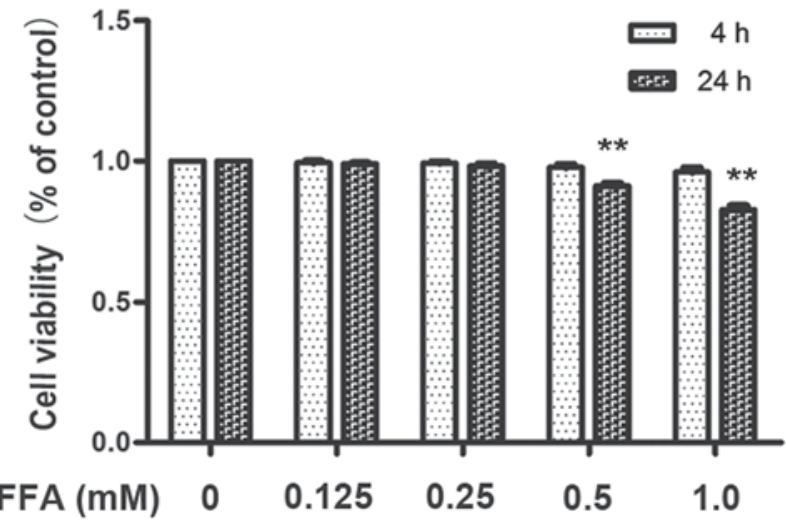

Figure 1. Cell viability of mature 3T3-L1 adipocytes pre-treated with FFA $(0,0.125,0.25,0.5,1.0 \mathrm{mM})$ for $4 \mathrm{~h}$ or $24 \mathrm{~h}$ was determined by MTT assay. Representative results were from three independent experiments. ${ }^{* *} \mathrm{P}<0.01$, vs. control group.

insulin resistance and plays an important role in energy metabolism (20). Among the known NO synthases, eNOS was originally identified as playing an important role in the regulation of vascular tone and blood pressure. However, eNOS expression is not restricted to vascular endothelium and has been shown to be more ubiquitous. eNOS has a major role in adiponectin synthesis of adipocytes (21). In present study, we determined the effects of FFAs on NO production and eNOS expression of 3T3-L1 adipocytes in the presence or absence of Rb1. The results showed that the levels of eNOS expression (Fig. 3A) and NO production (Fig. 3B) were very low in control cells. FFA significantly decreased eNOS expression and the corresponding NO production that were significantly restored by $40 \mu \mathrm{M}$ Rb1 (Fig. 3).

FFA decreases SOD2 expression and increases ROS generation in 3T3-L1 adipocytes, and these effects are reversed by $R b 1$. SOD2 is a major anti-oxidant enzyme in mitochondria, which catalyzes the dismutation of $\mathrm{O}_{2}$ into $\mathrm{H}_{2} \mathrm{O}_{2}$, and is one of the Nrf2-regulated SODs. To compensate for the lack of $\mathrm{NO}$ bioavailability and reduce $\mathrm{O}_{2}$-mediated damage, SOD increases $\mathrm{H}_{2} \mathrm{O}_{2}$ levels by dismutation of superoxide anions (22). In this study, cultured 3T3-L1 adipocytes were exposed to $0.5 \mathrm{mM}$ FFAs in the absence or presence of Rb1 at 10, 20 and $40 \mu \mathrm{m}$ for $4 \mathrm{~h}$. ROS generation as well as SOD2 expression were then measured. The results showed that FFA treatment for 4 h drastically decreased SOD2 expression (Fig. 4A), and increased ROS levels (Fig. 4B) in 3T3-L1 adipocytes, which were restored by $\mathrm{Rb} 1$ in a dose-dependent manner.

$N F-\kappa B$ activation in 3T3-L1 adipocytes is induced by FFA, and the effect is inhibited by $R b 1$. NF- $\mathrm{B}$-dependent pathways are important to regulate inflammatory gene expression in adipocytes. In the inactive state, $\mathrm{NF}-\kappa \mathrm{B} / \mathrm{Rel}$ transcription factors are present in the cytosol. However, when cells are stimulated by stress factors, the NF- $\mathrm{NB}$ p65 subunit is phosphorylated and initiates the inflammatory response. In the present study, we examined the effect of Rb1 on phosphorylation of the NF- $\kappa$ B p65 subunit at Ser536. The results showed that FFAs $(0.5 \mathrm{mmol} / \mathrm{l}, 4 \mathrm{~h})$ induced phosphorylation of the NF- $\kappa \mathrm{B}$ p65 subunit at Ser536, and Rb1 pretreatment (10, 20, $40 \mu \mathrm{M})$ 

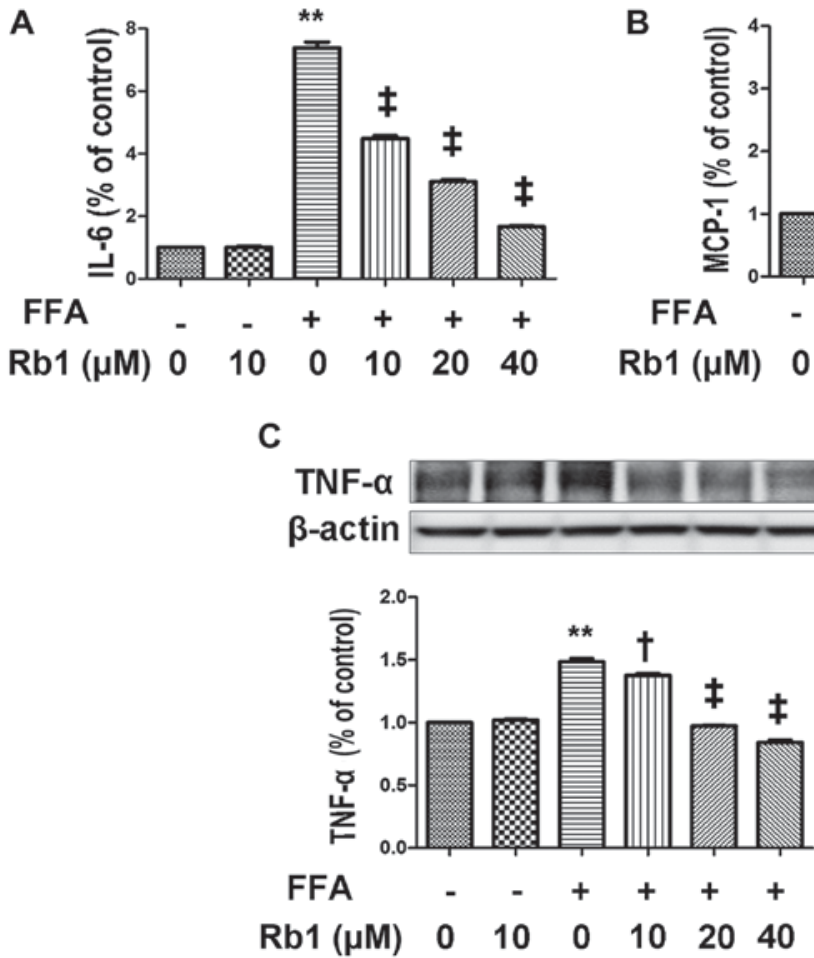

Figure 2. Rb1 suppressed FFA-induced IL-6, MCP-1 secretion, and TNF- $\alpha$ expression in 3T3-L1 adipocytes. Saturated palmitic acid was used as FFA in this study. Fully differentiated 3T3-L1 adipocytes were cultured for $16 \mathrm{~h}$ in DMEM with $2 \%$ FBS and were preincubated with Rb1 at 10,20 , and $40 \mu \mathrm{M}$ for $4 \mathrm{~h}$ followed by exposure to $0.5 \mathrm{mM}$ FFA or FFA-free BSA for $4 \mathrm{~h}$ respectively. Saturated palmitic acid was used in this study. (A) IL-6 and (B) MCP-1 secreted into the medium were measured by ELISAs and normalized by cell number. (C) Expression of TNF- $\alpha$ was tested by western blot analysis and semi-quantified by densitometry. Representative results were from three independent experiments. ${ }^{* *} \mathrm{P}<0.01$, vs. control group; ${ }^{\dagger} \mathrm{P}<0.05$, vs. FFA group; ${ }^{\ddagger} \mathrm{P}<0.01$, vs. FFA group.
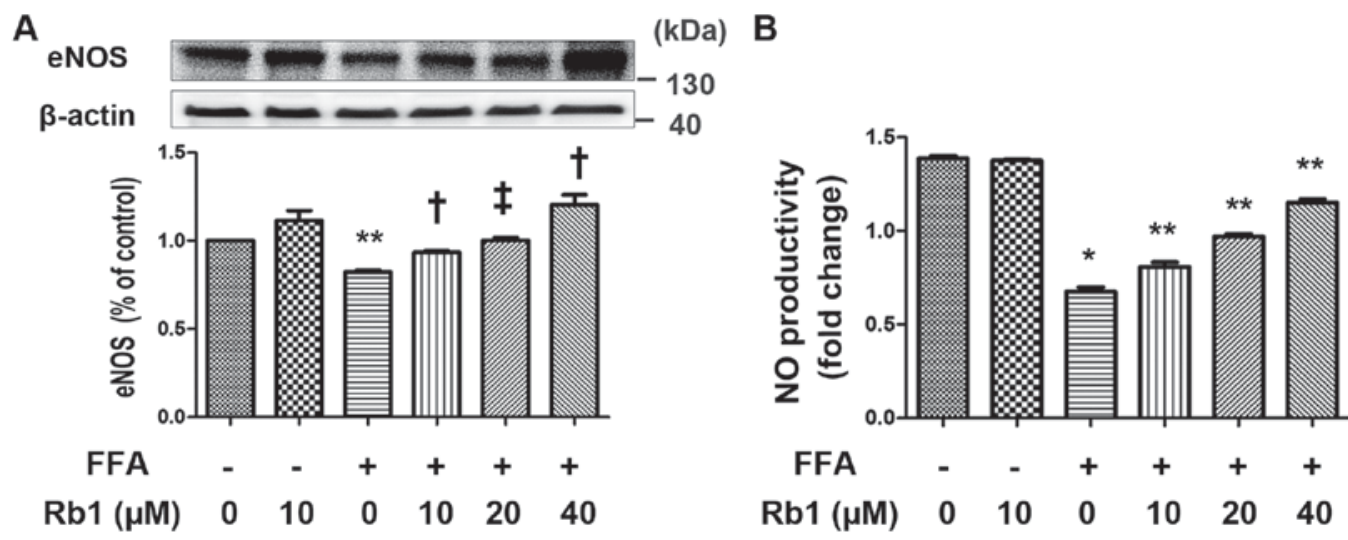

Figure 3. Rb1 reversed FFA-induced eNOS protein downregulation, and consequently increased NO production. Saturated palmitic acid was used as FFA in this study. Fully differentiated 3T3-L1 adipocytes were cultured for $16 \mathrm{~h}$ in DMEM with 2\% FBS and were preincubated with Rb1 at 10, 20 , and $40 \mu \mathrm{M}$ for $4 \mathrm{~h}$ followed by exposure to $0.5 \mathrm{mM}$ FFA or FFA-free BSA for $4 \mathrm{~h}$ respectively. Saturated palmitic acid was used in this study. (A) Expression of eNOS was determined by western blot analysis. (B) NO production was measured by Griess reaction and expressed as percentage of control. Representative results were from three independent experiments. ${ }^{*} \mathrm{P}<0.05$, vs. control group; ${ }^{* * *} \mathrm{P}<0.01$, vs. control group; ${ }^{\dagger} \mathrm{P}<0.05$, vs. FFA group; ${ }^{*} \mathrm{P}<0.01$, vs. FFA group.

reduced phosphorylation of the NF-kB p65 subunit at Ser536 in a dose-dependent manner $(\mathrm{P}<0.01)$ (Fig. 5). However, total NF-кB p65 in 3T3-L1 adipocytes was unchanged (Fig. 5).

\section{Discussion}

Past extensive studies have identified that Rb1 protects various cell types from injuries by anti-inflammatory and anti-oxidant functions. Cheng et al confirmed that Rb1 suppresses the
IL-1 $\beta$-induced inflammatory response and apoptosis in human articular chondrocytes (23). Xia et al demonstrated that $\mathrm{Rb} 1$ inhibits myocardial ischemia/reperfusion injury in diabetic rats by enhancing eNOS expression (24). Moreover, $\mathrm{Rb} 1$ functions as an anti-diabetic factor by improving central leptin sensitivity (25), increasing basal glucose uptake, and promoting browning by improving PPAR- $\gamma$ activity (26). Interestingly, direct evidence concerning the role of $\mathrm{Rb} 1$ in FFA-induced oxidative stress and inflammatory responses in 
A

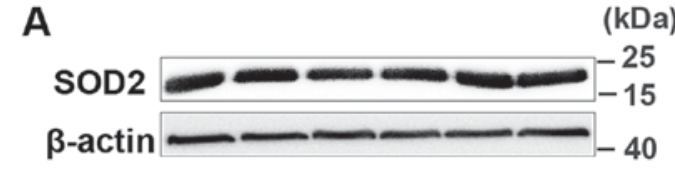

B
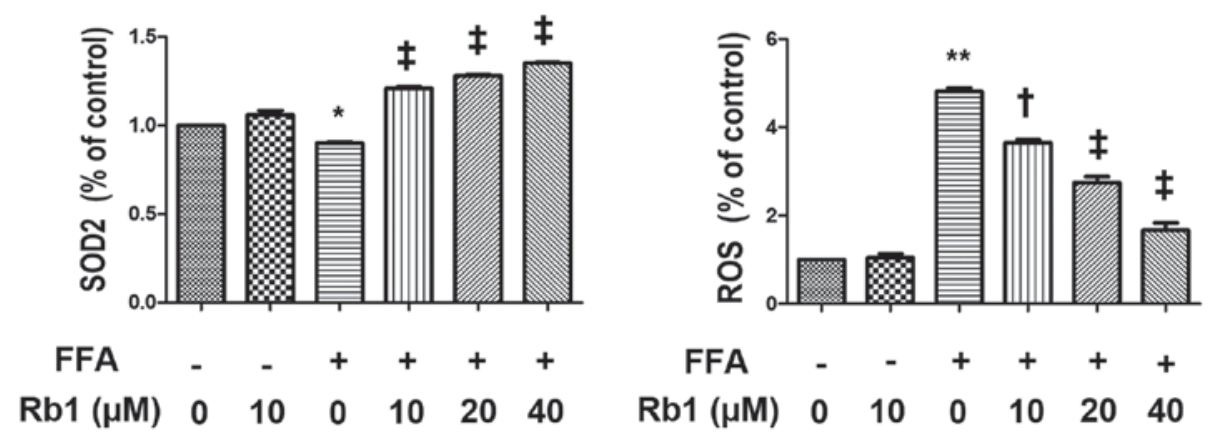

Figure 4. Rb1 counteracted FFA-induced SOD2 protein downregulation and ROS generation. Saturated palmitic acid was used as FFA in this study. Fully differentiated 3T3-L1 adipocytes were cultured for $16 \mathrm{~h}$ in DMEM with $2 \%$ FBS and were preincubated with Rb1 at 10,20 , and $40 \mu \mathrm{M}$ for $4 \mathrm{~h}$ followed by exposure to $0.5 \mathrm{mM}$ FFA or FFA-free BSA for $4 \mathrm{~h}$ respectively. Saturated palmitic acid was used in this study. (A) Expression of SOD2 was determined by western blot analysis. (B) ROS levels were measured by DCFH-DA and expressed as percentage of control. Representative results were from three independent experiments. ${ }^{*} \mathrm{P}<0.05$, vs. control group; ${ }^{* *} \mathrm{P}<0.01$, vs. control group; ${ }^{\dagger} \mathrm{P}<0.05$, vs. FFA group; ${ }^{*} \mathrm{P}<0.01$, vs. FFA group.
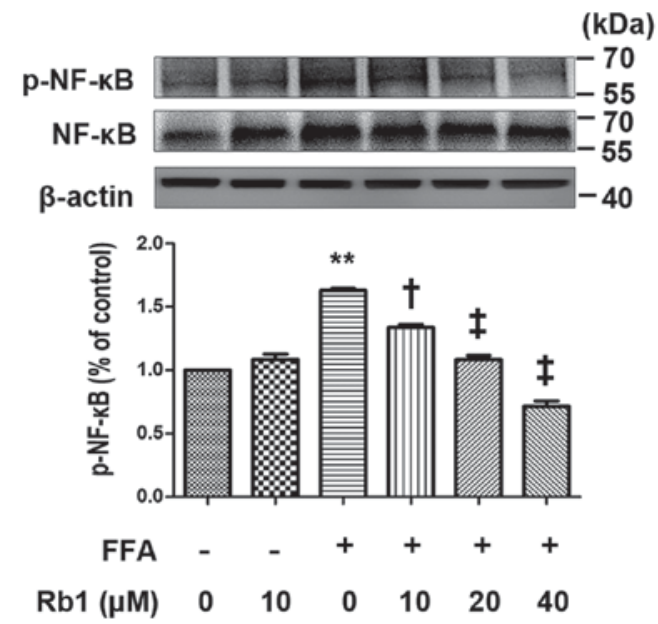

Figure 5. Rb1 inhibited FFA-induced phosphorylation of NF- $\kappa \mathrm{B}$ p65 protein in 3T3-L1 adipocytes. Saturated palmitic acid was used as FFA in this study. Fully differentiated 3T3-L1 adipocytes were cultured for $16 \mathrm{~h}$ in DMEM with $2 \%$ FBS and were preincubated with $\mathrm{Rb} 1$ at 10,20 , and $40 \mu \mathrm{M}$ for $4 \mathrm{~h}$ followed by exposure to $0.5 \mathrm{mM}$ FFA or FFA-free BSA for $4 \mathrm{~h}$ respectively. Phosphorylation of NF- $\kappa$ B p65 subunit at Ser536 was determined by western blot analysis. Representative results were from three independent experiments. ${ }^{* *} \mathrm{P}<0.01$, vs. control group; ${ }^{\dagger} \mathrm{P}<0.05$, vs. FFA group; ${ }^{\dagger} \mathrm{P}<0.01$, vs. FFA group.

3T3-L1 adipocytes has not been fully elucidated. Our previous study showed that Rb1 protects HUVECs from senescence by modulation of eNOS activity (27) and stimulation of the Sirtuin-1 pathway (16). In the present study, we demonstrated that $0.5 \mathrm{mM}$ FFAs decreased NO production and increased ROS generation that subsequently activated the $\mathrm{NF}-\kappa \mathrm{B}$ pathway, leading to overproduction of IL-6, MCP-1, and TNF- $\alpha$ in 3T3-L1 adipocytes. Rb1 ameliorates oxidative stress by increasing SOD2 and eNOS expression and suppressing $\mathrm{NF}-\kappa \mathrm{B}$ activation, and thus reduces IL-6, MCP-1, and TNF- $\alpha$ production. The inhibitory effect of Rb1 on pro-inflammatory cytokine production contributes to its beneficial anti-obesity and anti-diabetic effects.
Circulating pro-inflammatory cytokines from adipocytes to their downstream sensors in liver, muscle, etc. lead to insulin resistance. Moreover, previous studies have demonstrated that excessive circulating levels of FFAs are released from ectopic fat deposits, which activate TNF- $\alpha$, protein kinase C, c-Jun NH2-terminal kinase, JNK1, and IL-6 expression (28-30) in the state of obesity. The concentrations of FFAs in vivo range from 0.1 to $1 \mathrm{mM}(31,32)$. Therefore, we chose an FFA concentration of $0.5 \mathrm{mM}$ for our studies to reflect a conservative estimate of the in vivo conditions. We also confirm that the saturated FFA palmitate is an effective inducer of the inflammatory response in 3T3-L1 adipocytes, which is consistent with the study by Kolapo and associates (33).

The NF- $\kappa \mathrm{B}$ pathway is a classical inflammation signaling pathway. $N F-\kappa B$ is activated by a variety of stimuli and plays a critical role in the regulation of multiple cytokines such as TNF- $\alpha$, MCP-1, and IL-6 (34,35). Previous studies have demonstrated that FFA is a potent inducer of $\mathrm{NF}-\kappa \mathrm{B}$ activation in monocyte/macrophages $(36,37)$. In our study, we demonstrated that FFAs upregulated phosphorylation of the $\mathrm{NF}-\kappa \mathrm{B}$ p65 subunit at Ser536, which is in consistent with the result of previous studies $(33,38,39)$. In addition, our findings were in agreement with those showing that palmitate induced secretions of MCP-1 and IL- 6 as well as TNF- $\alpha$ expressions in 3T3-L1 adipocytes $(39,40)$. Furthermore, we showed that Rb1 inhibited palmitate-induced NF- $\kappa$ B p65 phosphorylation and release of pro-inflammatory cytokines. Few literatures have reported the effect of Rb1 on FFA treated 3T3-L1 adipocytes and mechanisms. Wang et al (41) demonstrated that Rb1 attenuated intestinal injury by inhibiting the $\mathrm{NF}-\kappa \mathrm{B}$ activation and induced inflammatory cytokines in the lung tissues. Cheng and associates showed that Rb1 inhibited osteoclast genesis by modulating NF- $\kappa \mathrm{B}$ pathway (42). These results are consistent with our findings and highlight possible protective mechanism of anti-inflammatory effects of Rb1. However, phosphorylation of $\mathrm{NF}-\kappa \mathrm{B}$ does not represent direct evidence of subsequent DNA binding, phosphorylation of NF- $\kappa \mathrm{B}$ is an essential step for subsequent DNA binding. Further experiments investigating 
the mechanism of Rb1 in phosphorylation at cellular or animal levels should be conducted in the future.

Oxidative stress is also closely associated with the development of obesity and diabetes $(43,44)$, which is characterized by decreased expression of anti-oxidant genes, such as eNOS and SOD2, overproduction of ROS, and less NO generation. There are many conflicting studies on NO production during oxidative stress. Previous reports have shown that NO is associated with the initiation and maintenance of inflammation through the generation of peroxynitrite in human inflammatory bowel disease (45), which can result in decreased cellular insulin sensitivity by causing inhibitory nitrosylation of Akt (46). Conversely, Kashyap et al showed that impaired NO activity might play an important role in the insulin resistance of type 2 diabetic individuals, indicating that an NO-dependent increase is an important mediator of insulin-stimulated glucose disposal in insulin target tissues (47). These conflicting results may be caused by different cell types and different time courses. In the present study, we found that FFAs decreased NO production in 3T3-L1 adipocytes, whereas Rb1 treatment attenuated this effect. eNOS plays a major role in adipocytes metabolism. Nisoli et al demonstrated $\mathrm{eNOS}^{-/-}$mice showed features of insulin resistance (48). Koh and associates showed that plasma adiponectin concentrations were reduced in adult $\mathrm{eNOS}^{-/}$mice compared with age-matched wild-type mice (21). In our study, expression of eNOS was upregulated by Rb1 in FFA-treated 3T3-L1 adipocytes in a dose-dependent manner followed by increased NO production in 3T3-L1 adipocytes.

Mitochondria metabolize oxygen and is a major source of ROS. One outcome of excessive levels of ROS is modification of the structure and function of adipocytes and lipids, leading to adipocyte dysfunction including altered cell signaling, impaired energy metabolism, and inflammation (42). SODs are considered as antioxidant defense enzymes that catalyze the conversion of two superoxides into hydrogen peroxide and oxygen. Yeop et al demonstrated that treatment with the antioxidants, $\mathrm{N}$-acetyl cysteine, catalase and SOD repressed ROS generation and $\mathrm{NF}-\kappa \mathrm{B}$ translocation stimulated by excess glucose and palmitate, and decreased inflammatory gene expression (40). A decreased level and activity of SODs can result in the accumulation of superoxide anion radicals in cell and induction of SOD2 is suggested to protect against excess ROS (49). To study the role of SOD2 in obesity, Krautbauer and associates treated 3T3-L1 preadipocytes or mature adipocytes with increasing concentrations of palmitate (PA), oleate (OA) or linoleate (LA) (from 0 to $200 \mu \mathrm{M}$ ). They demonstrated that SOD2 is induced not only in visceral adipose tissues of rodents fed a high diet but also induced by increased concentrations of FFA in mature 3T3-L1 adipocyte in contrast to premature adipocytes. In addition, they showed that OA $(200 \mu \mathrm{M})$ upregulated SOD2 during day 6 and day 9 in mature 3T3-L1 adipocytes (50). Wang and associates identified that SOD2 expression had been markedly increased as well as mitochondrial DNA content in visceral fat (VF) of C57BL/6J mice fed a high-fat and high-sucrose diet (HFHSD) at 6th month, while a further extension of HFHSD diet intervention resulted in a decrease of mitochondrial biogenesis and SOD2 expression in the VF until to the 12th month (51). The findings of a recent study by Kang and colleagues suggested that heterozygous SOD2 deletion impaired glucose-stimulated insulin secretion in high-fat-fed (HF) mice (52). Moreover, the study of Bauer et al showed that elevated free fatty acids and impaired adiponectin bioactivity contribute to reduced SOD2 protein in monocytes of type 2 diabetes patients (53). These findings argue that whether increased concentrations of FFA decrease SOD2 expression, and whether SOD2 has beneficial or deleterious effects on obesity and insulin sensitivity. However, the effect of palmitate to SOD2 in mature adipocyte have not been reported in their paper. Our result showed that palmitate at the concentration of $0.5 \mathrm{mM}$ suppressed SOD2 protein expression in mature 3T3-L1 adipocytes. Rb1 has been shown to up-regulate the activity of SODs and enhance expression of hypoxia-inducible factor- $1 \alpha$ in hepatic tissues in previous studies (54). Furthermore, recent studies showed that pretreatment with Rb1 significantly protects various cell types against oxidative injury and upregulates Nrf2 and its downstream antioxidant-responsive genes including SOD2 $(55,56)$. In our study, we found that Rb1 increased the expression of SOD2, which was consistent with the diminished production of ROS. It has been clearly indicated that Rb1 protects 3T3-L1 adipocytes from FFA-induced redox stress, which is in line with previous investigations (57).

Taken together, our study demonstrates that pretreatment with Rb1 ameliorates pro-inflammatory cytokine expression through suppressed NF- $\kappa \mathrm{B}$ translocation and blockade of its activation. The inhibitory effect of Rb1 on oxidative stress is attributed to its anti-inflammatory activity and anti-oxidative functions, and thus may contribute to the anti-obesity effect of $\mathrm{Rb} 1$ in insulin resistance and diabetes. The lack of animal and clinical data is a limitation of our study, but it provides an important basis for future research.

\section{Acknowledgements}

This work was supported by the grants from the National Natural Science Foundation of China (grant number: 81300707, to Min Wang; 81370447, to Xiaoxian Qian); The funders had no role in study design, data collection and analysis, decision to publish, or preparation of the manuscript. We thank Mitchell Arico for critical proofreading and editing of the manuscript.

\section{References}

1. Lau DC, Dhillon B, Yan H, Szmitko PE and Verma S: Adipokines: Molecular links between obesity and atheroslcerosis. Am J Physiol Heart Circ Physiol 288: H2031-H2041, 2005.

2. Guilherme A, Virbasius JV, Puri V and Czech MP: Adipocyte dysfunctions linking obesity to insulin resistance and type 2 diabetes. Nat Rev Mol Cell Biol 9: 367-377, 2008.

3. Schwartz B and Yehuda-Shnaidman E: Putative role of adipose tissue in growth and metabolism of colon cancer cells. Front Oncol 4: 164, 2014.

4. Grant RW and Dixit VD: Adipose tissue as an immunological organ. Obesity (Silver Spring) 23: 512-518, 2015.

5. Harwood HJ Jr: The adipocyte as an endocrine organ in the regulation of metabolic homeostasis. Neuropharmacology 63: 57-75, 2012.

6. Mraz M and Haluzik M: The role of adipose tissue immune cells in obesity and low-grade inflammation. J Endocrinol 222: R113-R127, 2014.

7. Wellen KE and Hotamisligil GS: Obesity-induced inflammatory changes in adipose tissue. J Clin Invest 112: 1785-1788, 2003.

8. Zhang W, Mottillo EP, Zhao J, Gartung A, VanHecke GC, Lee JF, Maddipati KR, Xu H, Ahn YH, Proia RL, et al: Adipocyte lipolysis-stimulated interleukin- 6 production requires sphingosine kinase 1 activity. J Biol Chem 289: 32178-32185, 2014. 
9. Boden G: Obesity and free fatty acids. Endocrinol Metab Clin North Am 37: 635-646, viii-ix, 2008.

10. Inoguchi T, Li P, Umeda F, Yu HY, Kakimoto M, Imamura M, Aoki T, Etoh T, Hashimoto T, Naruse M, et al: High glucose level and free fatty acid stimulate reactive oxygen species production through protein kinase $\mathrm{C}$-dependent activation of NAD (P)H oxidase in cultured vascular cells. Diabetes 49: 1939-1945, 2000.

11. Dasgupta S, Bhattacharya S, Biswas A, Majumdar SS Mukhopadhyay S, Ray S and Bhattacharya S: NF-kappaB mediates lipid-induced fetuin-A expression in hepatocytes that impairs adipocyte function effecting insulin resistance. Biochem J 429: 451-462, 2010.

12. Dong X, Zheng L, Lu S and Yang Y: Neuroprotective effects of pretreatment of ginsenoside Rb1 on severe cerebral ischemia-induced injuries in aged mice: Involvement of anti-oxidant signaling. Geriatr Gerontol Int 17: 338-345, 2017.

13. Fu Y and Ji LL: Chronic ginseng consumption attenuates age-associated oxidative stress in rats. J Nutr 133: 3603-3609, 2003.

14. Yu X, Ye L, Zhang H, Zhao J, Wang G, Guo C and Shang W: Ginsenoside $\mathrm{Rb} 1$ ameliorates liver fat accumulation by upregulating perilipin expression in adipose tissue of $\mathrm{db} / \mathrm{db}$ obese mice. J Ginseng Res 39: 199-205, 2015.

15. Xiong Y, Shen L, Liu KJ, Tso P, Xiong Y, Wang G, Woods SC and Liu M: Antiobesity and antihyperglycemic effects of ginsenoside Rb1 in rats. Diabetes 59: 2505-2512, 2010

16. Song Z, Liu Y, Hao B, Yu S, Zhang H, Liu D, Zhou B, Wu L, Wang M, Xiong Z, et al: Ginsenoside Rb1 prevents $\mathrm{H} 2 \mathrm{O} 2$-induced HUVEC senescence by stimulating sirtuin-1 pathway. PLoS One 9: e112699, 2014.

17. Kratchmarova I, Kalume DE, Blagoev B, Scherer PE, Podtelejnikov AV, Molina H, Bickel PE, Andersen JS, Fernandez MM, Bunkenborg J, et al: A proteomic approach for identification of secreted proteins during the differentiation of 3T3-L1 preadipocytes to adipocytes. Mol Cell Proteomics 1: 213-222, 2002

18. Wang M, Wang JJ, Li J, Park K, Qian X, Ma JX and Zhang SX: Pigment epithelium-derived factor suppresses adipogenesis via inhibition of the MAPK/ERK pathway in 3T3-L1 preadipocytes. Am J Physiol Endocrinol Metab 297: E1378-E1387, 2009.

19. Svedberg J, Björntorp P, Smith U and Lonnroth P: Free-fatty acid inhibition of insulin binding, degradation and action in isolated rat hepatocytes. Diabetes 39: 570-574, 1990.

20. Sugita H, Kaneki M, Tokunaga E, Sugita M, Koike C, Yasuhara S, Tompkins RG and Martyn JA: Inducible nitric oxide synthase plays a role in LPS-induced hyperglycemia and insulin resistance. Am J Physiol Endocrinol Metab 282: E386-E394, 2002

21. Koh EH, Kim M, Ranjan KC, Kim HS, Park HS, Oh KS, Park IS Lee WJ, Kim MS, Park JY, et al: eNOS plays a major role in adiponectin synthesis in adipocytes. Am J Physiol Endocrinol Metab 298: E846-E853, 2010.

22. Thomas SR, Chen K and Keaney JF Jr: Hydrogen peroxide activates endothelial nitric-oxide synthase through coordinated phosphorylation and dephosphorylation via a phosphoinositide 3-kinase-dependent signaling pathway. J Biol Chem 277: 6017-6024, 2002.

23. Cheng W, Wu D, Zuo Q, Wang Z and Fan W: Ginsenoside Rb1 prevents interleukin-1 beta induced inflammation and apoptosis in human articular chondrocytes. Int Orthop 37: 2065-2070, 2013.

24. Xia R, Zhao B, Wu Y, Hou JB, Zhang L, Xu JJ and Xia ZY: Ginsenoside Rb1 preconditioning enhances eNOS expression and attenuates myocardial ischemia/reperfusion injury in diabetic rats. J Biomed Biotechnol 2011: 767930, 2011.

25. Wu Y, Yu Y, Szabo A, Han M and Huang XF: Central inflammation and leptin resistance are attenuated by ginsenoside Rb1 treatment in obese mice fed a high-fat diet. PLoS One 9: e92618, 2014.

26. Mu Q, Fang X, Li X, Zhao D, Mo F, Jiang G, Yu N, Zhang Y, Guo Y, Fu M, et al: Ginsenoside Rb1 promotes browning through regulation of PPARgamma in 3T3-L1 adipocytes. Biochem Biophys Res Commun 466: 530-535, 2015.

27. Liu DH, Chen YM, Liu Y, Hao BS, Zhou B, Wu L, Wang M, Chen L, Wu WK and Qian XX: Ginsenoside Rb1 reverses $\mathrm{H} 2 \mathrm{O} 2$-induced senescence in human umbilical endothelial cells: Involvement of eNOS pathway. J Cardiovasc Pharmacol 59: 222-230, 2012
28. Neacsu O, Cleveland K, Xu H, Tchkonia TT, Kirkland JL and Boney CM: IGF-I attenuates FFA-induced activation of JNK1 phosphorylation and TNFalpha expression in human subcutaneous preadipocytes. Obesity (Silver Spring) 21: 1843-1849, 2013.

29. Chiadak JD, Arsenijevic T, Verstrepen K, Gregoire F, Bolaky N, Delforge V, Flamand V, Perret J and Delporte C: Forskolin inhibits lipopolysaccharide-induced modulation of MCP-1 and GPR120 in 3T3-L1 adipocytes through an Inhibition of NFкB. Mediators Inflamm 2016: 1431789, 2016.

30. Jiao P, Chen Q, Shah S, Du J, Tao B, Tzameli I, Yan W and Xu H: Obesity-related upregulation of monocyte chemotactic factors in adipocytes: Involvement of nuclear factor-kappaB and c-Jun NH2-terminal kinase pathways. Diabetes 58: 104-115, 2009.

31. Laine PS, Schwartz EA, Wang Y, Zhang WY, Karnik SK, Musi N and Reaven PD: Palmitic acid induces IP-10 expression in human macrophages via NF-kappaB activation. Biochem Biophys Res Commun 358: 150-155, 2007

32. Jiao P, Ma J, Feng B, Zhang H, Diehl JA, Chin YE, Yan W and $\mathrm{Xu}$ H: FFA-induced adipocyte inflammation and insulin resistance: Involvement of ER stress and IKK $\beta$ pathways. Obesity (Silver Spring) 19: 483-491, 2011

33. Ajuwon KM and Spurlock ME: Palmitate activates the NF-kappaB transcription factor and induces IL-6 and TNFalpha expression in 3T3-L1 adipocytes. J Nutr 135: 1841-1846, 2005.

34. Zhang WJ and Frei B: Astragaloside IV inhibits NF- $\kappa$ B activation and inflammatory gene expression in LPS-treated mice. Mediators Inflamm 2015: 274314, 2015.

35. Ye J and Keller JN: Regulation of energy metabolism by inflammation: a feedback response in obesity and calorie restriction. Aging (Albany NY) 2: 361-368, 2010.

36. Yang Z, Kahn BB, Shi H and Xue BZ: Macrophage alpha1 AMP-activated protein kinase (alpha1AMPK) antagonizes fatty acid-induced inflammation through SIRT1. J Biol Chem 285: 19051-19059, 2010.

37. Shi H, Kokoeva MV, Inouye K, Tzameli I, Yin H and Flier JS TLR4 links innate immunity and fatty acid-induced insulin resistance. J Clin Invest 116: 3015-3025, 2006.

38. Sun J, Luo J, Ruan Y, Xiu L, Fang B, Zhang H, Wang M and Chen $\mathrm{H}$ : Free fatty acids activate renin-angiotensin system in 3T3-L1 adipocytes through nuclear factor-kappa B pathway. J Diabetes Res 2016: 1587594, 2016.

39. McCall KD, Holliday D, Dickerson E, Wallace B, Schwartz AL Schwartz C, Lewis CJ, Kohn LD and Schwartz FL: Phenylmethimazole blocks palmitate-mediated induction of inflammatory cytokine pathways in 3T3L1 adipocytes and RAW 264.7 macrophages. J Endocrinol 207: 343-353, 2010.

40. Yeop Han C, Kargi AY, Omer M, Chan CK, Wabitsch M, O'Brien KD, Wight TN and Chait A: Differential effect of saturated and unsaturated free fatty acids on the generation of monocyte adhesion and chemotactic factors by adipocytes: Dissociation of adipocyte hypertrophy from inflammation. Diabetes 59: 386-396, 2010

41. Wang J, Qiao L, Li S and Yang G: Protective effect of ginsenoside Rb1 against lung injury induced by intestinal ischemia-reperfusion in rats. Molecules 18: 1214-1226, 2013.

42. Cheng B, Li J, Du J, Lv X, Weng L and Ling C: Ginsenoside Rb1 inhibits osteoclastogenesis by modulating NF- $\mathrm{KB}$ and MAPK pathways. Food Chem Toxicol 50: 1610-1615, 2012

43. Gerber PA and Rutter GA: The role of oxidative stress and hypoxia in pancreatic beta-cell dysfunction in diabetes mellitus. Antioxid Redox Signal 26: 501-518, 2017.

44. Chang YC and Chuang LM: The role of oxidative stress in the pathogenesis of type 2 diabetes: From molecular mechanism to clinical implication. Am J Transl Res 2: 316-331, 2010.

45. Kolios G, Valatas V and Ward SG: Nitric oxide in inflammatory bowel disease: A universal messenger in an unsolved puzzle. Immunology 113: 427-437, 2004.

46. Yasukawa T, Tokunaga E, Ota H, Sugita H, Martyn JA and Kaneki M: S-nitrosylation-dependent inactivation of Akt/protein kinase B in insulin resistance. J Biol Chem 280: 7511-7518, 2005.

47. Kashyap SR, Roman LJ, Lamont J, Masters BS, Bajaj M, Suraamornkul S, Belfort R, Berria R, Kellogg DL Jr, Liu Y and DeFronzo RA: Insulin resistance is associated with impaired nitric oxide synthase activity in skeletal muscle of type 2 diabetic subjects. J Clin Endocrinol Metab 90: 1100-1105, 2005.

48. Nisoli E, Clementi E, Paolucci C, Cozzi V, Tonello C, Sciorati C, Bracale R, Valerio A. Francolini M, Moncada S and Carruba MO: Mitochondrial biogenesis in mammals: The role of endogenous nitric oxide. Science 299: 896-899, 2003 
49. Ikegami Y, Inukai K, Imai K, Sakamoto Y, Katagiri H, Kurihara S, Awata T and Katayama S: Adiponectin upregulates ferritin heavy chain in skeletal muscle cells. Diabetes 58: 61-70, 2009.

50. Krautbauer S, Eisinger K, Neumeier M, Hader Y, Buettner R, Schmid PM, Aslanidis C and Buechler C: Free fatty acids, lipopolysaccharide and IL-1alpha induce adipocyte manganese superoxide dismutase which is increased in visceral adipose tissues of obese rodents. PLoS One 9: e86866, 2014.

51. Wang PW, Kuo HM, Huang HT, Chang AY, Weng SW, Tai MH Chuang JH, Chen IY, Huang SC, Lin TK and Liou CW: Biphasic response of mitochondrial biogenesis to oxidative stress in visceral fat of diet-induced obesity mice. Antioxid Redox Signal 20: 2572-2588, 2014.

52. Kang L, Dai C, Lustig ME, Bonner JS, Mayes WH, Mokshagundam S, James FD, Thompson CS, Lin CT, Perry CG, et al: Heterozygous SOD2 deletion impairs glucose-stimulated insulin secretion, but not insulin action, in high-fat-fed mice. Diabetes 63: 3699-3710, 2014.
53. Bauer S, Wanninger J, Neumeier M, Wurm S, Weigert J, Kopp A, Bala M, Schäffler A, Aslanidis C and Buechler C: Elevated free fatty acids and impaired adiponectin bioactivity contribute to reduced SOD2 protein in monocytes of type 2 diabetes patients. Exp Mol Pathol 90: 101-106, 2011.

54. Guo Y, Yang T, Lu J, Li S, Wan L, Long D, Li Q, Feng L and Li Y: $\mathrm{Rb} 1$ postconditioning attenuates liver warm ischemia-reperfusion injury through ROS-NO-HIF pathway. Life Sci 88: 598-605, 2011.

55. Ye J, Yao JP, Wang X, Zheng M, Li P, He C, Wan JB, Yao X and $\mathrm{Su} \mathrm{H}$ : Neuroprotective effects of ginsenosides on neural progenitor cells against oxidative injury. Mol Med Rep 13: 3083-3091, 2016.

56. Fan J, Liu D, He C, Li X and He F: Inhibiting adhesion events by Panax notoginseng saponins and Ginsenoside Rb1 protecting arteries via activation of Nrf2 and suppression of p38-VCAM-1 signal pathway. J Ethnopharmacol 192: 423-430, 2016.

57. Liu DH, Chen YM, Liu Y, Hao BS, Zhou B, Wu L, Wang M, Chen L, Wu WK and Qian XX: Rb1 protects endothelial cells from hydrogen peroxide-induced cell senescence by modulating redox status. Biol Pharm Bull 34: 1072-1077, 2011. 